\title{
MR defecography detects pelvic floor dysfunction in participants with chronic complete spinal cord injury
}

\author{
Cornelia Putz ${ }^{1}$ Celine D. Alt ${ }^{2,4} \cdot$ Bjoern Wagner $^{1} \cdot$ Simone Gantz ${ }^{3} \cdot$ Hans J. Gerner $\mathbb{D}^{1} \cdot$ Norbert Weidner $^{1} \cdot$ \\ Lars Grenacher $^{2,5} \cdot$ Cornelia Hensel $^{1}$
}

Received: 15 November 2018 / Revised: 21 July 2019 / Accepted: 22 July 2019 / Published online: 10 September 2019

(c) The Author(s), under exclusive licence to International Spinal Cord Society 2019

\begin{abstract}
Study design A prospective single arm study.

Objectives Previously we have demonstrated that magnetic resonance (MR) defecography is feasible in participants with complete spinal cord injury (SCI). The main aim of this study is to evaluate whether MR defecography can provide objective parameters correlating with the clinical manifestations of neurogenic bowel dysfunction (NBD) in participants with SCI. Setting A monocentric study in a comprehensive care university hospital Spinal Cord Injury Center.

Methods Previously published MR defecography parameters (anorectal angle (ARA), hiatal descent (M-line) and hiatal width (H-line)) of twenty participants with SCI were now compared to a standardized clinical assessment of NBD. Descriptive statistics, correlations and t-tests for independent samples were calculated.

Results The significantly higher values for the ARA at rest and M-line at rest in participants with SCI correlated with the clinical assessment of bowel incontinence. Furthermore, in nearly half of the investigated SCI cohort the normally positive difference between ARA, M-line and H-line at rest and during defecation became negative suggesting pelvic floor dyssynergia as a potential mechanism underlying constipation in people with complete SCI. In fact, these participants showed a more severe clinical presentation of NBD according to the total NBD score.

Conclusions MR defecography provides objective parameters correlating with clinical signs of NBD, such as constipation and bowel incontinence. Therefore, MR defecography can support pathophysiology-based decision-making with respect to specific therapeutic interventions, which should help to improve the management of NBD.
\end{abstract}

Cornelia Putz

corneliaputz0805@googlemail.com

1 Spinal Cord Injury Center, Heidelberg University Hospital, Schlierbacher Landstrasse 200a, 69118 Heidelberg, Germany

2 Department of Diagnostic and Interventional Radiology, University Hospital Heidelberg, Im Neuenheimer Feld 110, 69120 Heidelberg, Germany

3 Department of Experimental Orthopaedics, Heidelberg University Hospital, Schlierbacher Landstrasse 200a, 69118 Heidelberg, Germany

4 Present address: University Duesseldorf, Medical Faculty, Department of Diagnostic and Interventional Radiology, 40225 Duesseldorf, Germany

5 Present address: Diagnostic Clinic Munich, Department of Radiology and Nuclear Medicine, Augustenstraße 115, 80798 Muenchen, Germany

\section{Introduction}

Neurogenic bowel dysfunction (NBD) affects the majority of people with spinal cord injury (SCI) impairing social activities and quality of life [1,2]. Therefore, improving bladder and bowel function represents a highly prioritized therapeutic goal for people with SCI [3].

NBD is defined as dysfunction of the colon with clinical symptoms of constipation, fecal incontinence, and disordered defecation due to loss of normal sensory and/or motor control $[4,5]$. Clinical practical guidelines for neurogenic bowel management include conservative management such as diet, fluid intake, medication, and digital rectal stimulation to promote stool transit, induce defecation in a predictable time frame, minimize the occurrence of unexpected bowel events, and reduce gastrointestinal symptoms such as constipation or overdistension of the colon [6-9]. The time point and relevance of surgical options (e.g. colostomy) is still being debated [10]. With increasing time 
following injury gastrointestinal problems become more frequent [11-13]. In particular, constipation related problems such as time for each defecation and the use of laxatives increases, although the rate of fecal incontinence remains stable at $18 \%$ [11]. The occurrence of incontinence is higher in people with complete SCI in comparison to people with an incomplete injury, affecting the quality of life in $62 \%$ of all people with SCI $[14,15]$. Constipation is partially attributed to a moderately increased colon transit time and a lack of anal sphincter muscle relaxation [5]. The propulsive function of the colon can be estimated by determining total and segmental colon transit time using both, for example, radiopaque markers and clinical examination. The anal sphincter muscle can be assessed manually by anorectal manometry or otherwise with an electromyography [5]. These respective methods do not allow for visualization of anorectal function at rest or during defecation. In contrast, magnetic resonance (MR) defecography has been successfully employed to analyze the coordinated contraction of pelvic floor muscles in a dynamic way. Pathological changes in defined MR defecography parameters indicate uncoordinated contraction of pelvic floor muscles reflecting dyssynergic defecation, which can be observed in participants suffering from chronic constipation without underlying neurological disease conditions [16-18]. We have previously shown that MR defecography adapted to participants with chronic complete SCI characterized by a complete loss of voluntary anal contraction is feasible [19]. The MR-defecography procedure was modified by omitting imaging during squeezing (maximal voluntary sphincter contraction) and initiating bowel evacuation with suppositories due to the absence of voluntary pelvic floor/anal sphincter muscle control. The received MR defecography measurements (Fig. 1) in participants with SCI were compared to historical able-bodied control cohorts [20-23] and showed higher values for mean anorectal angle (ARA) and hiatal width (H-line) during rest and defecation [19].

The main aim of this study was to evaluate whether these parameters can detect uncoordinated pelvic floor/anal sphincter muscle contraction as a sequel of NBD in participants with chronic complete SCI. MR defecography derived parameters were correlated with clinical scores reflecting the severity and quality of NBD.

\section{Methods}

Following the MR defecography study (trial number S-274/ 2008) from March 2010 until April 2012 at the Spinal Cord Injury Center, Heidelberg University Hospital, Germany [19], the obtained parameters were now compared with clinical parameters.

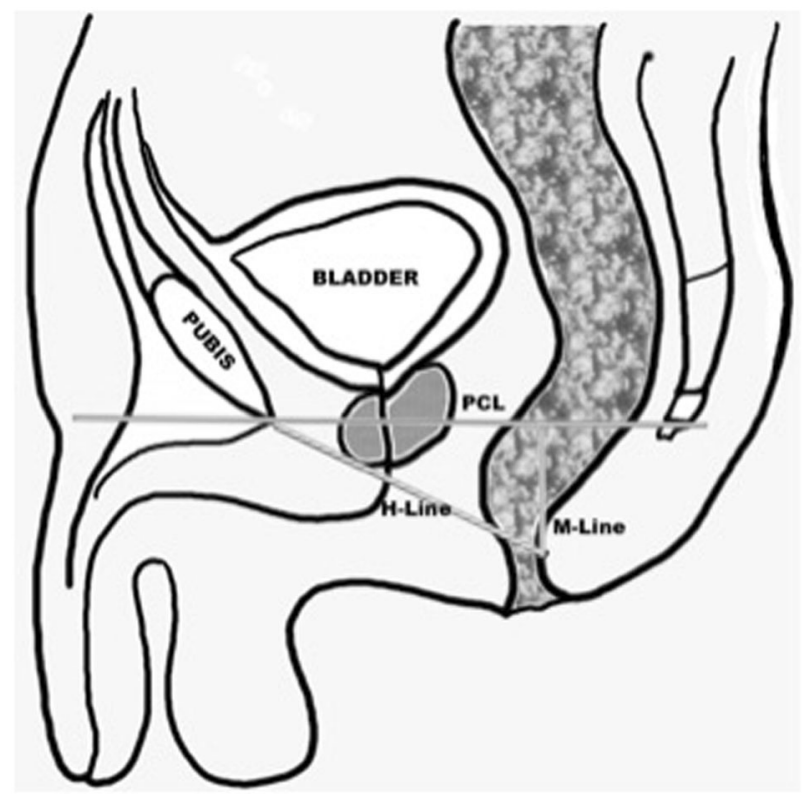

Fig. 1 Anatomical landmarks and MR-measurements (male participant): The PCL as the reference line is drawn from the inferior border of the pubis to the last visible joint of os coccygeus. The hiatal width (H-line) is measured from the inferior border of the pubic symphysis to the posterior aspect of the anorectum where the puborectal muscle forms a sling. From this endpoint, the M-Line (hiatal descent) is drawn perpendicular to the PCL to measure the descent of the levator plate to the level of the pelvic floor

\section{Participant population, inclusion and exclusion criteria}

A study population of 20 participants with a sensorimotor complete traumatic AIS-A (AIS-American Spinal Injury Association Impairment Scale) SCI with a neurological level of injury (NLI) between Th1 and Th10, which were previously investigated in the MR defecography feasibility study [19], were now compared with relevant clinical parameters. T10 was chosen as the most caudal NLI to exclude participants with additional or exclusive lower motoneuron damage in the conus region of the spinal cord. For detailed inclusion and exclusion criteria please see our previous study [19].

\section{Clinical parameters}

A study baseline of participants' specific data (age, time since injury, routine bowel evacuation practise) was collected. Each participant received a standardized neurological examination according to the International Standards for Neurological Classification of SCI (ISNCSCI) to confirm the NLI and lesion completeness [24]. All participants were asked to complete the NBD questionnaire, which contains 
items of colorectal and anal dysfunction [25]. In detail, the following 10 items were checked: (1) frequency of bowel movements $(0,1$, or 6 points), (2) duration of the defecation ( 0,3 or 7 points), (3) discomfort during defecation ( 0 or 2 points), (4) medication (tablets) against constipation (0 or 2 points), (5) medication (drops or liquids) against constipation (0 or 2 points), (6) use of digital stimulation (0 or 6 points), (7) frequency of fecal incontinence $(0,6,7$, or 13 points), (8) frequency of flatus incontinence ( 0 or 2 points), (9) medication against unexpected bowel movement ( 0 or 4 points), and (10) any perianal skin problems (0 or 3 points). Based on a total score of 47 points, participants are classified as having very minor (0-6), minor (7-9), moderate (10-13 points), or severe NBD (14-47 points). Each point value represents a weighted answer. For example, with respect to the frequency of fecal incontinence (item 7) the following four answers are possible: less than once a month (0 points); 1-4 times a month (6 points); $1-6$ times a week (7 points) and daily (13 points) [25].

\section{MR defecography parameters}

We used a modified MR-defecography procedure as described before [19, 26, 27]. Of note, in participants with sensorimotor complete SCI (AIS-A) images during squeezing (maximal voluntary sphincter contraction) and volitional evacuation cannot be obtained. A rectal catheter was positioned in the rectum and filled with aqueous sonographic gel to contrast the rectum and suppositories were inserted to initiate the defecation reflex. From the time point of rectal filling until evacuation dynamic sequences from a defined MR-imaging protocol were obtained in an endless loop. If an evacuation could not be observed within $15 \mathrm{~min}$ of imaging we repeated the rectal filling and performed another rectal stimulation. The acquired images were evaluated offline by an experienced radiologist, who was blinded to all clinical data. For the analysis we took two images of the pelvic floor: the first one at rest after filling the rectum with ultrasound gel (Img0) and the second one during notable defecation (Img1) (Fig. 2). Then the difference $(\Delta)$ was calculated for ARA, hiatal descent (M-line) and hiatal width of the levator plate (H-line) [19].

\section{Statistical analysis}

Statistical analysis was performed using IBM SPSS Statistics for Windows, Version 20.0. (Armonk, NY: IBM Corp) and $\mathrm{R}$ version 3.5.3. Basic descriptive statistics (mean and standard deviation), correlations and t-test for independent samples were conducted to compare the participants with each other. All tests were performed as two-tailed and the significance level was set conventionally at $p<0.05$.

\section{Results}

Previously obtained MR defecography results [19] were correlated with clinical parameters (participant characteristics and NBD-score) from 20 participants (18 men, 2 women), all with a traumatic sensorimotor complete SCI without sacral sparing (AIS-A). The NBD-score evaluates predominantly aspects of constipation and fecal incontinence in the context of NBD. According to the overall NBD score three participants suffer from very minor, two from minor, eight from moderate and seven from severe NBD. Based on the sub-item 7 "frequency of fecal incontinence" subgroups of participants with rare episodes of incontinence (less than once a month or 1-4 times a month) vs. frequent episodes of incontinence (daily or 1-6 times a week) were formed. Accordingly, 17 participants suffer from rare incontinence episodes ( $\leq 4$ times a month) and three from severe incontinence epiosodes $(\geq 1$ times per week).

Participants suffering from frequent incontinence episodes presented with significantly higher values for ARA at rest $(p=0,00018$; Fig. 3a) and for hiatal descent (M-line) at rest $(p=0,045$; Fig. 3 b) compared to participants with rare incontinence episodes. Subgrouping with respect to routine initiation of defecation (administration of suppositories, digital stimulation, digital evacuation, transanal irrigation) revealed a significantly lower hiatal width of the levator plate at rest $(\mathrm{H} 0)$ in participants applying digital stimulation ( $p=0,028$, Fig. 4).

In individuals without NBD each of the parameters analyzed (ARA, H-line/M-line length) physiologically increases from the measurement at rest to the measurement during defecation. Therefore, a negative delta $(\Delta)$ was considered as non-physiological, representing abnormal pelvic floor movement. Based upon this premise 9 out of the 20 participants with SCI displayed abnormal pelvic floor movement. One participant even revealed negative values for all three parameters $\Delta \mathrm{ARA}, \Delta \mathrm{H}$-line and $\Delta \mathrm{M}$-line. One to two negative parameters were observed in eight participants (Table 1). Participants with SCI identified to suffer from abnormal pelvic floor movement based upon negative values for $\Delta$ ARA, $\Delta \mathrm{H}$ or $\Delta \mathrm{M}$ showed a trend toward a more severe presentation of NBD expressed by a higher mean NBD score of $15( \pm 4 \mathrm{SD})$ compared to a NBD score of $11( \pm 5 \mathrm{SD})$ in all participants without any negative $\Delta$ values $(p=0.061)$. A NBD score of 15 would be classified as severe, whereas a score of 11 only be classified as moderate.

The other clinical parameters (e.g. age, time post-injury, NLI, routine bowel evacuation practise (suppositories, digital evacuation, transanal irrigation) showed no correlation with the obtained parameters. 

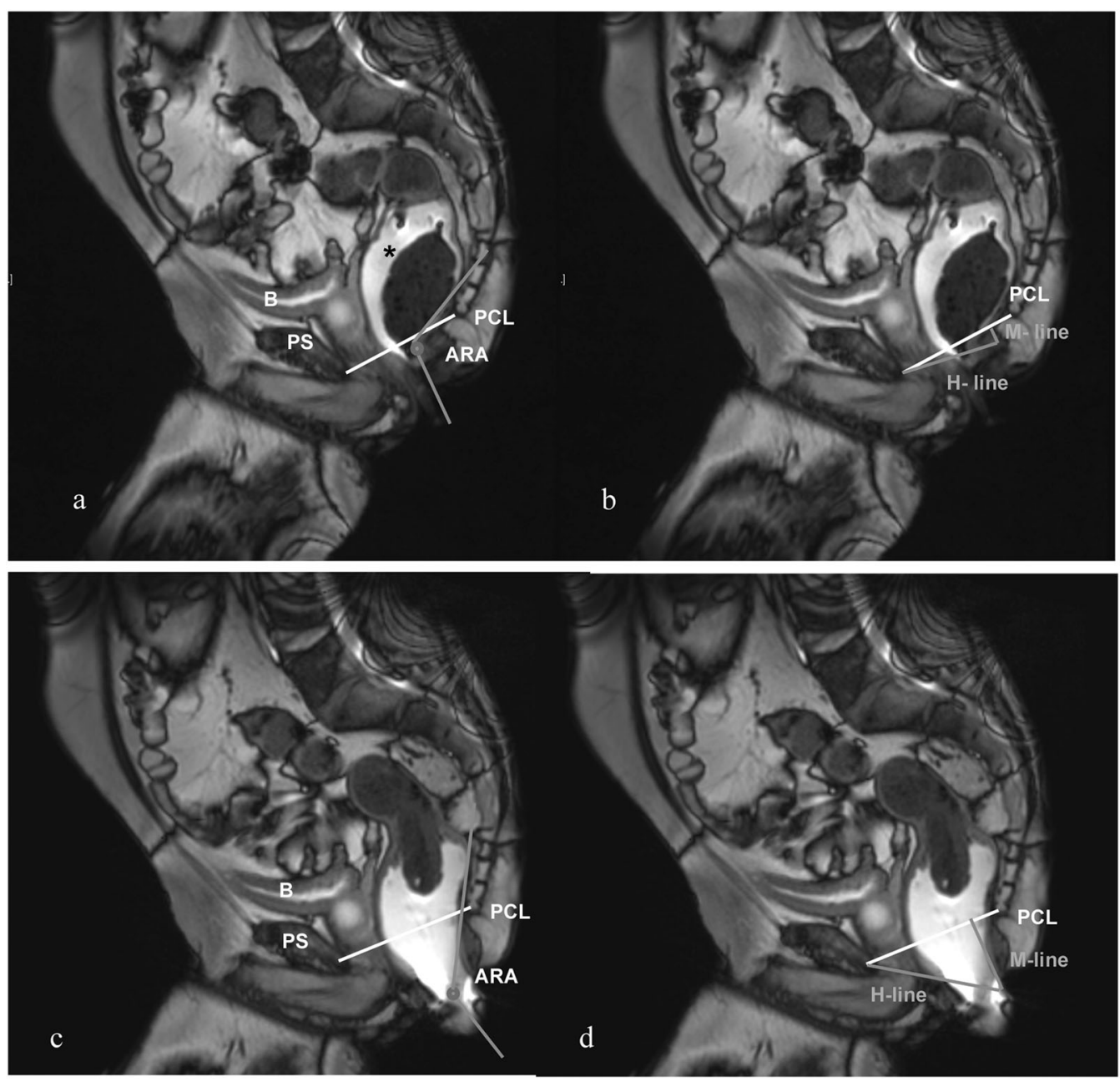

Fig. 2 Radiological parameters at rest (a and b) and during defecation (c and d) in a 45-year-old male participant with sensorimotor complete paraplegia (NLI T8) 11 years after injury with positive changes of $\Delta$ ARA, $\Delta$ M- line and $\Delta \mathrm{H}$ - line

Fig. 3 Fecal incontinence and MR defecography parameter. (a) ARA at rest (ARA 0 in $\left.^{\circ}\right)$ in participants with rare $(\leq 4$ times a month; $n=17)$ vs. frequent episodes of fecal incontinence ( $\geq 1$ times per week; $n=3$ ). (b) $\mathrm{M}$-line at rest $\left(\mathrm{M} 0\right.$ in $\left.{ }^{\circ}\right)$ in participants with rare $(\leq 4$ times a month; $n=17)$ vs. frequent episodes of fecal incontinence ( $\geq 1$ times per week; $n=3$ )
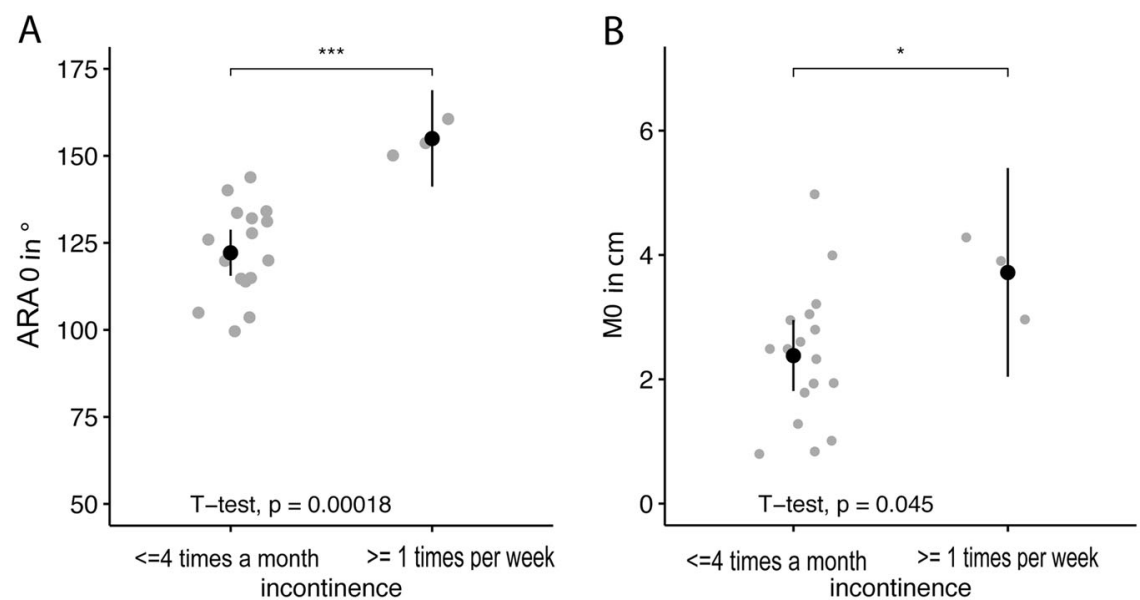


\section{Discussion}

Beyond the overall feasibility to employ MR defecography in people with SCI characterized by the complete loss of voluntary sphincter control [19], this non-invasive imaging technique is capable of detecting objective parameters, which correlate with major hallmarks of NBD, namely bowel incontinence and pelvic floor dyssynergia. The latter has been associated with the bowel constipation, a frequent clinical problem in people with SCI suffering from NBD.

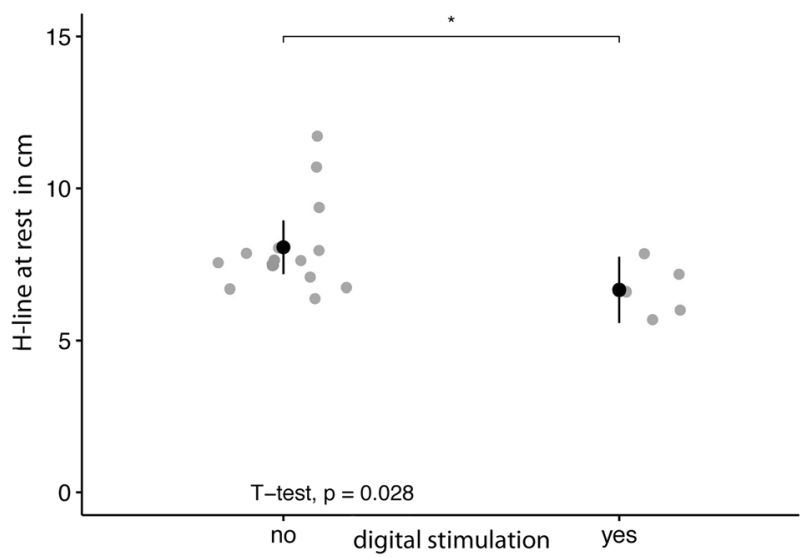

Fig. 4 Digital stimulation and MR defecography parameter. H-line at rest $(\mathrm{H} 0$ in $\mathrm{cm})$ in relation to digital stimulation
The presence of a negative delta values in any of the investigated measures (ARA, H-line/M-line length) during defecation minus respective measures at rest was determined as dyssynergic pelvic floor movement. In chronic constipation disorder, without any known underlying neurological disease condition, uncoordinated/dyssynergic defecation has been detected by MR defecography as a relevant mechanism underlying outlet obstruction [17]. In almost half of our study population the negative delta values indicated that the normally highly synchronized interaction of pelvic floor and anal sphincter muscles during rest and defecation becomes uncoordinated. Most likely, the loss of supraspinal control elicits uncoordinated pelvic floor/anal sphincter muscle activity reflecting spasticity, which causes dyssynergic defecation with the consequence of outlet obstruction and constipation [28, 29]. In this study, spasticity was not assessed with respective assessments (Ashworth, Spinal Cord Assessment Tool for Spastic ReflexesSCATS). Therefore, lower extremity spasticity could not be correlated with pathological MR defecography findings to address this hypothesis.

The employed NBD score suggests an overall correlation of clinically relevant NBD with the presence of a negative delta value in any of the investigated measures (ARA, Hline/M-line length) [25]. However, the NBD score does not allow one to unequivocally attribute pelvic floor dyssynergia to constipation. In subsequent studies a clinical score
Table 1 Clinical and radiological data of the study group of participants with sensorimotor complete SCI $(n=$ 20). 11 participants $(1-11)$ showed positive changes in all analyzed parameters $(\Delta \mathrm{ARA}$, $\Delta \mathrm{M}, \Delta \mathrm{H})$ and 9 showed at least one negative difference (highlighted in BOLD) in any parameter (12-20)

\begin{tabular}{|c|c|c|c|c|c|c|c|}
\hline Participant & Age & NBD & NLI & ARA at rest $\left(^{\circ}\right)$ & $\Delta \mathrm{ARA}\left({ }^{\circ}\right)$ & $\Delta \mathrm{M}(\mathrm{cm})$ & $\Delta \mathrm{H}(\mathrm{cm})$ \\
\hline 1 & 45 & 4 & 8 & 115.4 & 25.3 & 3.2 & 2.5 \\
\hline 2 & 59 & 12 & 6 & 120 & 17.2 & 4.5 & 2.3 \\
\hline 3 & 45 & 3 & 3 & 114 & 10.2 & 0.7 & 0.3 \\
\hline 4 & 24 & 12 & 4 & 105.3 & 18.6 & 1.6 & 0.1 \\
\hline 5 & 70 & 22 & 9 & 150.2 & 7.2 & 2.5 & 1.9 \\
\hline 6 & 40 & 9 & 4 & 144.3 & 7.1 & 0.2 & 0.3 \\
\hline 7 & 38 & 9 & 4 & 134.2 & 29.8 & 5 & 3.2 \\
\hline 8 & 51 & 14 & 6 & 100 & 28.8 & 2.1 & 0.9 \\
\hline 9 & 60 & 15 & 2 & 120.6 & 17 & 2.8 & 0.4 \\
\hline 10 & 47 & 13 & 9 & 126.7 & 7.1 & 3.1 & 1.4 \\
\hline 11 & 32 & 6 & 3 & 134 & 16.5 & 3.2 & 0.03 \\
\hline 12 & 43 & 11 & 10 & 115.5 & 4.2 & 0.1 & -1.3 \\
\hline 13 & 19 & 19 & 4 & 104 & 49.4 & -0.4 & 1.9 \\
\hline 14 & 47 & 23 & 8 & 154 & -23.5 & -0.4 & -0.9 \\
\hline 15 & 63 & 17 & 1 & 140.7 & -2.8 & 2.5 & 0.8 \\
\hline 16 & 54 & 11 & 3 & 130.6 & 9.8 & -0.7 & 0.6 \\
\hline 17 & 50 & 13 & 4 & 131.8 & -5.7 & -0.8 & 0.4 \\
\hline 18 & 54 & 12 & 3 & 115.2 & 15.9 & 0.1 & -4.6 \\
\hline 19 & 47 & 17 & 2 & 161.1 & -37 & 1.4 & -0.7 \\
\hline 20 & 45 & 13 & 3 & 127.6 & 11.2 & 1.2 & -0.6 \\
\hline
\end{tabular}

$N B D$-Score neurogenic bowel dysfunction score, $N L I$ neurological level of injury, $A R A$ anorectal angle, $H \mathrm{H}$ line, $M$ M-line, $\Delta$ difference between values at rest and defecation. 
depicting relevant aspects and severities of chronic constipation and fecal incontinence, which are the most relevant problem in chronic upper motoneuron type SCI, needs to be employed [30].

High values for the parameter ARA at rest and M-line at rest correlated with the presence of bowel incontinence according to the respective NBD score sub-item. MR defecography of subjects with fecal incontinence without underlying neurological disease indicated similar results with an increase of rectal descent of more than $6 \mathrm{~cm}$ and a wide ARA at rest [31]. Nevertheless, in people with SCI with an underlying UMN lesion and consecutive increased (pelvic floor) muscle tone, one would expect a rather low (less obtuse) ARA at rest. One possible reason for the flaccid pelvic floor could be peripheral neuropathy due to chronic pressure induced peripheral nerve damage [32]. In fact, in a high number of people with chronic SCI with underlying UMN lesions sensory nerve conduction studies of the sural nerve were negative, suggesting a chronic pressure lesion of sciatic nerves accelerated by increased pressure exposure due to frequent sitting and muscle atrophy in the lower back region [32]. In a similar manner pudendal nerves, which innervate both the external sphincter muscles as well as the puborectalis muscles, may have been harmed leading to secondary pelvic floor descent with increased (more obtuse) $A R A$ at rest and (longer) M-line at rest. Interestingly, the subgroup of SCI participants not using digital stimulation for bowel evacuation shows higher values for $\mathrm{H}$-line at rest. Under the assumption that digital stimulation was not performed due to the absence of respective rectal reflexes caused by respective muscle denervation, higher $\mathrm{H}$-line values in SCI people might indicate an underlying flaccid pelvic floor due to pudendal neuropathy. In this study, a digital rectal exam was only performed to exclude anorectal abnormalities and sacral sparing. However, anal reflexes, manometry or needle EMG of the anal sphincter to detect signs of muscle denervation or rectal pressure were not investigated here. Of course, respective findings have to be interpreted cautiously due to the rather low number of participants investigated. Only 3 out of 20 participants reported incontinence as a relevant problem. Nevertheless, the proportion of $15 \%$ fecal incontinence in this study matches the frequency of SCI related fecal incontinence in the literature [11].

In essence, the observed pelvic floor dyssynergia mirrors findings in neurogenic bladder dysfunction, in particular following UMN type SCI. The precisely coordinated sequence of bladder detrusor, internal and external sphincter muscle function is disrupted causing either urinary incontinence or urinary retention. For urinary retention various means of continuous or intermittent emptying of the bladder have been established, which dramatically help us to maintain patients' quality of life and to protect the urinary tract. In NBD, therapeutic measures are by far not as efficient. In people with SCI suffering from NBD presenting with chronic constipation, therapies aiming to increase the colon transit time (e.g. administration of laxatives, diet change, modified fluid intake) are recommended. However, pelvic floor dyssynergia with subsequent outlet obstruction leading to constipation cannot easily be addressed. If constipation cannot be solved sufficiently by above mentioned measures, more invasive therapeutic options may be considered to overcome outlet obstruction such as the introduction of irrigation systems, Malone antegrade continence enema, or colostomy [33]. In chronic constipation syndrome not related to neurogenic causes, botulinum toxin injections into the pelvic floor muscles have been performed in order to reduce muscle tone and thus overcome outlet obstruction [34], which could represent a therapeutic alternative in NBD related to SCI.

The main limitations of the study are the small sample size, the rather broad patient reported assessment tool (NBD score) and the absence of a healthy control group investigated in parallel. MR defecography, the way it was adapted to SCI subjects cannot be applied to able-bodied participants having voluntary anal contraction, which precludes healthy subjects as a control. The artificial interruption of anorectal sensorimotor function by means of superficial or invasive local anesthetic administration [35] does not allow one to create a more suitable control group. First of all, a major disruptive effect in terms of anorectal sensorimotor function was only achieved with invasive routes of local anesthetic administration, which would not receive ethics approval at our site. Secondly, with effective local anesthetic administration we would abolish defecation reflex loops, which are actually the main drivers of bowel movement in SCI patients with supraconal cord injuries during defecation. Specific characterization of upper (spasticity assessment) and lower (nerve conduction velocity, electromyography) motoneuron dysfunction beyond the pelvic floor is still required to get better insights into the mechanisms underlying the pathological changes observed with MR defecography. In line with many MRI quantification methods, the quantification of parameters (distances, angles) obtained from dynamic MRI contains the risk of measurement errors [36], which cannot be excluded in this study.

The study provides evidence that MR defecography can serve as a diagnostic tool, which would facilitate decision making with respect to more advanced therapeutic measures. However, at this point, while conclusive evidence is still absent, it is too early to recommend MR defecography as a standard diagnostic tool to assess NBD in people with SCI. Respective findings have to be confirmed in future studies, employing more sensitive clinical assessments with respect to constipation. Depending upon the results of such 
studies, pathophysiology driven specific therapeutic interventions beyond the commonly administered empirical treatment strategies can be envisioned.

\section{Data archiving}

Data were archieved in our local database.

Acknowledgements The authors thank Radhika Puttagunta for proof reading the manuscript.

Funding This study was financially supported by "Stiftung Orthopedic University Hospital Heidelberg".

Author Contributions Cornelia Putz, MD: Design, participant examinations, manuscript, analysis of data. Celine D Alt, MD: Evaluation of radiological parameters, correction of the manuscript. Bjoern Wagner, MD: Participant examinations, data acquisition. Simone Gantz: Statistic analysis. Hans J Gerner, MD: Design, correction of the manuscript. Nobert Weidner, MD: Analysis and interpretation of data, correction of the manuscript. Lars Grenacher, MD: Design, evaluation of radiological parameters, Cornelia Hensel, MD: Particpants examinations, analysis of data, manuscript.

\section{Compliance with ethical standards}

Statement of Ethics The MR defecography study including the clinical evaluation (trial number S-274/2008) has been proofed by the ethics committee Heidelberg University Hospital, Germany. We certify that all applicable institutional and governmental regulations concerning the ethical use of human volunteers were followed during the course of this study.

Conflict of interest The authors declare that they have no conflict of interest.

Publisher's note Springer Nature remains neutral with regard to jurisdictional claims in published maps and institutional affiliations.

\section{References}

1. Faaborg PM, Christensen P, Finnerup N, Laurberg S, Krogh K. The pattern of colorectal dysfunction changes with time since spinal cord injury. Spinal Cord. 2008;46:234-8.

2. Kirk PM, King RB, Temple R, Bourjaily J, Thomas P. Long-term follow-up of bowel management after spinal cord injury. SCI Nurs. 1997; 14:56-63.

3. Anderson KD. Targeting recovery: priorities of the spinal cordinjured population. J Neurotrauma. 2004;21:1371-83.

4. Chung EA, Emmanuel AV. Gastrointestinal symptoms related to autonomic dysfunction following spinal cord injury. Prog Brain Res. 2006;152:317-33.

5. Valles M, Vidal J, Clave P, Mearin F. Bowel dysfunction in patients with motor complete spinal cord injury: clinical, neurological, and pathophysiological associations. Am J Gastroenterol. 2006;101:2290-9.

6. Krassioukov A, Eng JJ, Claxton G, Sakakibara BM, Shum S. Neurogenic bowel management after spinal cord injury: a systematic review of the evidence. Spinal Cord. 2010;48:718-33.
7. Spinal Cord Medicine Consortium. Clinical practice guidelines: neurogenic bowel management in adults with spinal cord injury. $\mathrm{J}$ Spinal Cord Med. 1998;21:248-93.

8. Harari D, Minaker KL. Megacolon in patients with chronic spinal cord injury. Spinal Cord. 2000;38:331-9.

9. Harari D, Sarkarati M, Gurwitz JH, McGlinchey-Berroth G, Minaker KL. Constipation-related symptoms and bowel program concerning individuals with spinal cord injury. Spinal Cord. 1997;35:394-401.

10. Furlan JC, Urbach DR, Fehlings MG. Optimal treatment for severe neurogenic bowel dysfunction after chronic spinal cord injury: a decision analysis. Br J Surg. 2007;94:1139-50.

11. Nielsen SD, Faaborg PM, Finnerup NB, Christensen P, Krogh K. Ageing with neurogenic bowel dysfunction. Spinal Cord. 2017;55:769-73.

12. Stone JM, Nino-Murcia M, Wolfe VA, Perkash I. Chronic gastrointestinal problems in spinal cord injury patients: a prospective analysis. Am J Gastroenterol. 1990;85:1114-9.

13. Liu CW, Huang CC, Chen CH, Yang YH, Chen TW, Huang MH. Prediction of severe neurogenic bowel dysfunction in persons with spinal cord injury. Spinal Cord. 2010;48:554-9.

14. Lynch AC, Antony A, Dobbs BR, Frizelle FA. Bowel dysfunction following spinal cord injury. Spinal Cord. 2001;39:193-203.

15. Lynch AC, Wong C, Anthony A, Dobbs BR, Frizelle FA. Bowel dysfunction following spinal cord injury: a description of bowel function in a spinal cord-injured population and comparison with age and gender matched controls. Spinal Cord. 2000;38:717-23.

16. Piloni V, Tosi P, Vernelli M. MR-defecography in obstructed defecation syndrome (ODS): technique, diagnostic criteria and grading. Tech Coloproctol. 2013;17:501-10.

17. Reiner CS, Tutuian R, Solopova AE, Pohl D, Marincek B, Weishaupt D. MR defecography in patients with dyssynergic defecation: spectrum of imaging findings and diagnostic value. $\mathrm{Br}$ J Radiol. 2011;84:136-44.

18. Bharucha AE, Wald A, Enck P, Rao S. Functional anorectal disorders. Gastroenterology. 2006;130:1510-8.

19. Putz C, Alt CD, Hensel C, Wagner B, Gantz S, Gerner HJ, et al. 3T MR-defecography-A feasibility study in sensorimotor complete spinal cord injured patients with neurogenic bowel dysfunction. Eur J Radiol. 2017;91:15-21.

20. Weishaupt, DR \& Reiner, CS MR-Defäkographie aus Gastrointestinale MRT Theorie und Praxis, Herausgeber: M.G. Thomas C. Lauenstein. Wissenschaftsverlag (2009).

21. Schreyer AG, Paetzel C, Fürst A, Dendl LM, Hutzel E, MüllerWille R, et al. Dynamic magnetic resonance defecography in 10 asymptomatic volunteers. World J Gastroenterol. 2012; 18:6836-42.

22. Bharucha AE, Fletcher JG, Harper CM, Hough D, Daube JR, Stevens C, et al. Relationship between symptoms and disordered continence mechanisms in women with idiopathic faecal incontinence. Gut. 2005;54:546-55.

23. Comiter CV, Vasavada SP, Barbaric ZL, Gousse AE, Raz S. Grading pelvic prolapse and pelvic floor relaxation using dynamic magnetic resonance imaging. Urology. 1999;54:454-7.

24. Maynard FM Jr., Bracen MB, Creasey G, Ditunno JF Jr., Donovan WH, Ducker TB, et al. International Standards for Neurological and Functional Classification of Spinal Cord Injury. American Spinal Injury Association. Spinal Cord. 1997; 35:266-74.

25. Krogh K, Christensen P, Sabroe S, Laurberg S. Neurogenic bowel dysfunction score. Spinal Cord. 2006;44:625-31.

26. Pannu HK, Kaufmann HS, Cundiff GW, Gendary R, Bluemke DA, Fishman EK. Dynamic MR imaging of pelvic organ prolapse: spectrum of abnormalities. Radiographics. 2000;20:1567-82. 
27. Sprenger D, Lienemann A, Anthuber C, Reiser M. [Functional MRI of the pelvic floor: its normal anatomy and pathological findings]. Radiologe. 2000;40:451-7.

28. Kuijpers HC, Bleijenberg G. The spastic pelvic floor syndrome. A cause of constipation. Dis Colon Rectum. 1985;28:669-72.

29. Quint EH, Smith YR, Bowerman RA, DeLancey JO. Spasticity of the pelvic floor mimicking an obstructive anomaly. J Pediatr Adolesc Gynecol. 2003;16:83-85.

30. Altomare DF, Spazzafumo L, Rinaldi M, Dodi G, Ghiselli R, Piloni V. Set-up and statistical validation of a new scoring system for obstructed defaecation syndrome. Colorectal Dis. 2008; 10:84-88.

31. Bitti GT, Argiolas GA, Ballicu N, Caddeo E, Cecconi M, Demurtas G, et al. Pelvic floor failure: MR imaging evaluation of anatomic and functional abnormalities. RadioGraphics. 2014; $34: 429-48$.
32. Kamradt T, Rasch C, Schuld C, Böttinger M, Mürle B, Hensel C, et al. Spinal cord injury: association with axonal peripheral neuropathy in severely paralysed limbs. Eur J Neurol. 2013;20:843-8.

33. Hughes M. Bowel management in spinal cord injury patients. Clin Colon Rectal Surg. 2014;27:113-5.

34. Jost WH, Schrank B, Herold A, Leiss O. Functional outlet obstruction: anismus, spastic pelvic floor syndrome, and dyscoordination of the voluntary sphincter muscles. Definition, diagnosis, and treatment from the neurologic point of view. Scand J Gastroenterol. 1999;34:449-53.

35. Broens PM, Penninckx FM, Ochoa JB. Fecal continence revisited: the anal external sphincter continence reflex. Dis Colon Rectum. 2013;56:1273-81.

36. Morren GL, et al. Triphasic MRI of pelvic organ descent: sources of measurement error. Eur J Radiol. 2005;54:276-83. 